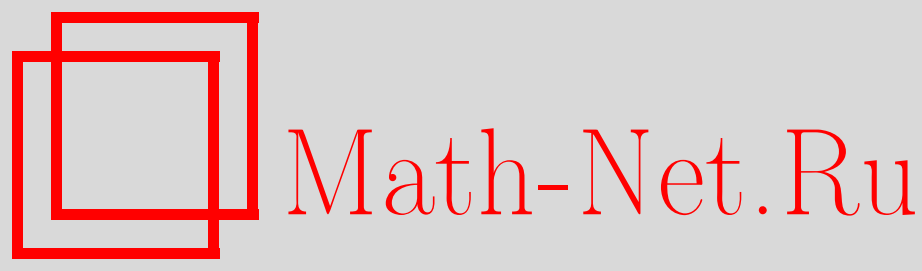

А. А. Хамзин, А. С. Никитин, А. С. Ситдиков, Д. А. Роганов, Осцилляции момента инерции конечной фермисистемы в рамках кренкинг-модели, ТМФ, 2013, том 176, номер 3, 458-476

DOI: https://doi.org/10.4213/tmf8446

Использование Общероссийского математического портала Math-Net.Ru подразумевает, что вы прочитали и согласны с пользовательским соглашением http: //www.mathnet.ru/rus/agreement

Параметры загрузки:

IP : 54.157 .27 .8

26 апреля 2023 г., 07:20:11

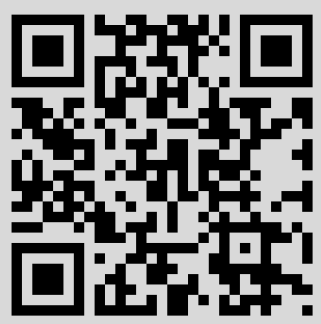




\title{
ОСЦИЛЛЯЦИИ МОМЕНТА ИНЕРЦИИ КОНЕЧНОЙ ФЕРМИ-СИСТЕМЫ В РАМКАХ КРЕНКИНГ-МОДЕЛИ
}

\begin{abstract}
В рамках кренкинг-модели с потенциалом анизотропного гармонического осциллятора проведен строгий аналитический расчет зависимости момента инерции конечной ферми-системы при конечных температурах от химического потенциала в адиабатическом пределе. Показано, что данная зависимость включает гладкую и осциллирующую составляющие. Найдены аналитические выражения для этих составляющих при произвольных температурах и частотах аксиальной деформации. Показано, что осцилляции момента инерции усиливаются при приближении к сферическому пределу и экспоненциально убывают с увеличением температуры.
\end{abstract}

Ключевые слова: конечная ферми-система, модель принудительного вращения, момент инерции ядра, анизотропный квантовый гармонический осциллятор, преобразование Меллина.

DOI: $10.4213 / \operatorname{tmf} 8446$

\section{1. ВВЕДЕНИЕ}

Коллективные вращения ядер успешно описываются в рамках нескольких теоретических подходов, которые главным образом основаны на модели принудительного вращения или кренкинг-модели [1]-[5]. Было показано [4], [5], что моменты инерции могут быть представлены в виде суммы гладкой классической твердотельной составляющей расширенного приближения Томаса-Ферми [6]-[8] и оболочечных поправок [4], полученных в рамках метода оболочечных поправок [9], [10], адаптированного к проблеме вращения. Точные аналитические решения для произвольной частоты вращения были получены для потенциала гармонического осциллятора [11] и распространены на конечные температуры [12], [13].

* Казанский (Приволжский) федеральный университет, Институт физики, Казань, Россия. E-mail: airat.khamzin@rambler.ru

${ }^{\dagger}$ Казанский государственный энергетический университет, Казань, Россия. E-mail: drnikitin@rambler.ru, airat_vm@rambler.ru

‡Акционерный инвестиционный коммерческий Банк “Татфондбанк”, Казань, Россия. E-mail: dima@e-inet.ru 
Следующим шагом в этом направлении стало развитие так называемой квазиклассической теории периодических орбит [6], [14], [15], которая стала одним из мощных и плодотворных инструментов для глубокого понимания и аналитического анализа основных особенностей оболочечной структуры в конечных вращающихся ферми-системах. Эта методика была применена в работах [13], [16], [17] для вычисления оболочечных поправок к моментам инерции конечных ферми-систем с деформированным потенциалом гармонического осциллятора, в этих работах также было проведено сравнение квазиклассических расчетов с квантово-механическими вычислениями. Были выявлены осцилляции в поведении момента инерции в зависимости от химического потенциала при фиксированных значениях параметра деформации. Было замечено, что при переходе к сферическому пределу амплитуды таких осцилляций существенно увеличиваются.

Целью настоящей работы является детальный аналитический расчет осцилляционной зависимости момента инерции конечной ферми-системы при конечных температурах от химического потенциала в рамках квантово-механических вычислений. Аналитическое исследование квантово-механического выражения для момента инерции в зависимости от температуры, числа частиц и деформации не проводилось в указанных работах [12], [13].

\section{2. КРЕНКИНГ-МОДЕЛЬ ДЛЯ ЯДЕРНОГО ВРАЩЕНИЯ И ЕЕ РЕШЕНИЯ ДЛЯ КОНЕЧНЫХ ТЕМПЕРАТУР}

В рамках кренкинг-модели вращение ядра вокруг оси $x$, перпендикулярной оси симметрии $z$ аксиально-симметричного потенциала $V(\mathbf{r})$ среднего поля, может быть описано путем решения задачи на собственные значения одночастичного гамильтониана во вращающейся системе координат, связанной с ядром [4], [5]:

$$
\widehat{H}^{\omega}=\widehat{H}_{0}-\omega \widehat{L}_{x}, \quad \widehat{H}_{0}=\sum_{i} \hat{h}_{0 i}, \quad \hat{h}_{0}=\frac{\hat{p}_{x}^{2}+\hat{p}_{y}^{2}+\hat{p}_{z}^{2}}{2 m}+V(\mathbf{r}),
$$

где $\omega$ - частота вращения, $\widehat{L}_{x}=\sum_{i} \hat{l}_{x i}$ - оператор проекции полного углового момента ядра на ось вращения, $\hat{l}_{x}-x$-компонента одночастичного углового момента. В случае, когда потенциал среднего поля равен потенциалу анизотропного гармонического осциллятора

$$
V(\mathbf{r})=\frac{m}{2}\left(\omega_{x}^{2} x^{2}+\omega_{y}^{2} y^{2}+\omega_{z}^{2} z^{2}\right),
$$

гамильтониан (1) легко диагонализуется и его собственные значения имеют вид [11]

$$
\varepsilon_{\alpha}^{\omega}=\hbar \omega_{x}\left(n_{x}+\frac{1}{2}\right)+\hbar \omega_{1}\left(n_{1}+\frac{1}{2}\right)+\hbar \omega_{2}\left(n_{2}+\frac{1}{2}\right),
$$

где

$$
\omega_{1,2}^{2}=\frac{1}{2}\left(\omega_{y}^{2}+\omega_{z}^{2}+2 \omega^{2}\right) \pm \frac{1}{2} \sqrt{\left(\omega_{y}^{2}-\omega_{z}^{2}\right)^{2}+8 \omega^{2}\left(\omega_{y}^{2}+\omega_{z}^{2}\right)},
$$

$\alpha=\left(n_{x}, n_{1}, n_{2}\right), n_{x, 1,2}$ - число квантов в соответствующих направлениях. Зная собственные значения и собственные функции $|\alpha\rangle=\left|n_{x} n_{1} n_{2}\right\rangle$ гамильтониана (1), 
несложно найти выражения для среднего значения одночастичного оператора проекции углового момента и момента инерции:

$$
\begin{aligned}
\left\langle\alpha\left|l_{x}\right| \alpha\right\rangle & =\hbar \omega\left\{\frac{2\left(\omega_{y}^{2}+\omega_{z}^{2}\right)}{\omega_{1}^{2}-\omega_{2}^{2}}\left(\frac{n_{2}+1 / 2}{\omega_{2}}-\frac{n_{1}+1 / 2}{\omega_{1}}\right)-\left(\frac{n_{2}+1 / 2}{\omega_{2}}+\frac{n_{1}+1 / 2}{\omega_{1}}\right)\right\}, \\
\Theta_{x}(\omega) & =\sum_{\alpha} \frac{\left\langle\alpha\left|l_{x}\right| \alpha\right\rangle}{\omega}=\hbar\left[\frac{2\left(\omega_{y}^{2}+\omega_{z}^{2}\right)}{\omega_{1}^{2}-\omega_{2}^{2}}-1\right] \frac{N_{2}}{\omega_{2}}-\hbar\left[\frac{2\left(\omega_{y}^{2}+\omega_{z}^{2}\right)}{\omega_{1}^{2}-\omega_{2}^{2}}+1\right] \frac{N_{1}}{\omega_{1}},
\end{aligned}
$$

где $N_{1,2}=\sum_{\alpha}\left(n_{1,2}+1 / 2\right)$ - общее число квантов в направлениях 1,2 [11]. Формула (6) для момента инерции известна в литературе как формула Зелевинского [11].

Естественным продолжением развития теории момента инерции системы нуклонов в деформированном потенциале гармонического осциллятора является обобщение формулы Зелевинского на конечные температуры. В работе [12] с использованием большого канонического распределения получена система уравнений для определения параметров формы ядра и момента инерции как функции температуры и числа частиц, которая исследовалась численными методами. В рамках численных расчетов анализировалась равновесная форма ядра в зависимости от температуры и числа частиц для случаев присутствия и отсутствия вращения. Во вращающейся системе координат при низких температурах обнаружена серия резких деформационных переходов, которые тесно коррелируют с оболочечной структурой гармонического осциллятора.

Ввиду исключительной важности этой формулы ниже мы продемонстрируем вывод момента инерции системы нуклонов, описываемой гамильтонианом (1), для конечных температур.

Воспользуемся большим термодинамическим потенциалом

$$
\Omega=-T \ln Z,
$$

соответствующим статистической сумме

$$
Z=\operatorname{Sp}\left(e^{-\left(\widehat{H}^{\omega}-\lambda \widehat{N}\right) / T}\right),
$$

где $T$ - температура в энергетических единицах, $\lambda$ - химический потенциал. В этом ансамбле числа заполнения одночастичных состояний подчиняются статистике Ферми, поэтому для $\Omega$ справедливо выражение

$$
\Omega=-T \sum_{\alpha} \ln \left(1+e^{-\left(\varepsilon_{\alpha}^{\omega}-\lambda\right) / T}\right),
$$

где энергии одночастичных состояний $\varepsilon_{\alpha}^{\omega}$ определяются выражением (3). Определим среднее значение полного углового момента $\widehat{L}_{x}=\sum_{i} \hat{l}_{x i}$ :

$$
\left\langle\widehat{L}_{x}\right\rangle=\frac{1}{Z} \operatorname{Sp}\left(e^{-\left(\widehat{H}^{\omega}-\lambda \widehat{N}\right) / T} \widehat{L}_{x}\right)=-\frac{\partial \Omega}{\partial \omega} .
$$

Воспользуемся выражениями для большого термодинамического потенциала (7) и статистической суммы (8), получим

$$
\left\langle\widehat{L}_{x}\right\rangle=-\sum_{\alpha} f\left(\varepsilon_{\alpha}^{\omega}-\lambda\right) \frac{\partial \varepsilon_{\alpha}^{\omega}}{\partial \omega},
$$


где $f(x)=\left(e^{x / T}+1\right)^{-1}-$ функция Ферми. Подставляя выражение $(3)$ для энергий одночастичных состояний $\varepsilon_{\alpha}^{\omega}$ и выполняя дифференцирование по $\omega$, найдем следующее выражение для момента инерции рассматриваемой модели при конечных температурах:

$$
\Theta_{x}=\frac{\left\langle\widehat{L}_{x}\right\rangle}{\omega}=\hbar\left\{\left[\frac{2\left(\omega_{y}^{2}+\omega_{z}^{2}\right)}{\omega_{1}^{2}-\omega_{2}^{2}}-1\right] \frac{N_{2}}{\omega_{2}}-\left[\frac{2\left(\omega_{y}^{2}+\omega_{z}^{2}\right)}{\omega_{1}^{2}-\omega_{2}^{2}}+1\right] \frac{N_{1}}{\omega_{1}}\right\}
$$

где $N_{1,2}$ определяются по формуле

$$
N_{1,2}=\sum_{\alpha} f\left(\varepsilon_{\alpha}^{\omega}-\lambda\right)\left(n_{1,2}+\frac{1}{2}\right)
$$

Полученное выражение для момента инерции по виду совпадает с выражением Зелевинского (6), но полное число квантов в направлениях 1, 2 определяются теперь по формулам (13).

В настоящем изложении мы сосредоточимся на анализе момента инерции в адиабатическом пределе, когда $\omega \rightarrow 0$, и для частного случая аксиальной симметрии: $\omega_{x}=\omega_{y} \equiv \omega_{\perp}$. В этом случае выражение (12) для момента инерции примет вид формулы Инглиса [1]

$$
\begin{aligned}
\Theta_{x} & =\frac{\hbar}{\omega_{\perp} \omega_{z}}\left\{\frac{\left(\omega_{z}-\omega_{\perp}\right)^{2}}{\omega_{\perp}+\omega_{z}}\left(N_{y}+N_{z}\right)+\frac{\left(\omega_{z}+\omega_{\perp}\right)^{2}}{\omega_{\perp}-\omega_{z}}\left(N_{z}-N_{y}\right)\right\}, \\
N_{y, z} & =\sum_{\alpha} f\left(\varepsilon_{\alpha}-\lambda\right)\left(n_{y, z}+\frac{1}{2}\right), \quad \alpha=\left(n_{x}, n_{y}, n_{z}\right) .
\end{aligned}
$$

Здесь $\varepsilon_{\alpha}=\hbar \omega_{\perp}\left(n_{x}+n_{y}+1\right)+\hbar \omega_{z}\left(n_{z}+1 / 2\right)$. Принимая во внимание условие сохранения потенциального объема $\omega_{x} \omega_{y} \omega_{z}=\omega_{0}^{3}$, где $\omega_{0}$ играет роль масштабного параметра, который будем считать не зависящим от температуры, удобно ввести параметр деформации $\eta=\omega_{\perp} / \omega_{z}>1$, через который выражаются частоты деформации

$$
\omega_{\perp}=\omega_{0} \eta^{1 / 3}, \quad \omega_{z}=\omega_{0} \eta^{-2 / 3} .
$$

Используя эти определения и вводя новые функции $N_{ \pm}=N_{z} \pm N_{y}$, приведем выражение для момента инерции (14) к следующему виду:

$$
\Theta_{x}=\hbar \frac{(\eta-1)^{3} N_{+}+(\eta+1)^{3} N_{-}}{\omega_{\perp}\left(\eta^{2}-1\right)} .
$$

С учетом аксиальной симметрии потенциала для дальнейших расчетов энергии одночастичных состояний удобно представить в виде

$$
\varepsilon_{n_{\perp} n_{z}}=\hbar \omega_{\perp}\left(n_{\perp}+\frac{n_{z}}{\eta}\right)+\varepsilon_{0}, \quad \varepsilon_{0}=\hbar \omega_{\perp}\left(1+\frac{1}{2 \eta}\right),
$$

а функции $N_{ \pm}=N_{z} \pm N_{y}$ в виде

$$
N_{ \pm}=\sum_{n_{\perp}, n_{z}} f\left(\varepsilon_{n_{\perp} n_{z}}-\lambda\right)\left(n_{\perp}+1\right)\left(n_{z}+\frac{1}{2} \pm \frac{n_{\perp}+1}{2}\right) .
$$



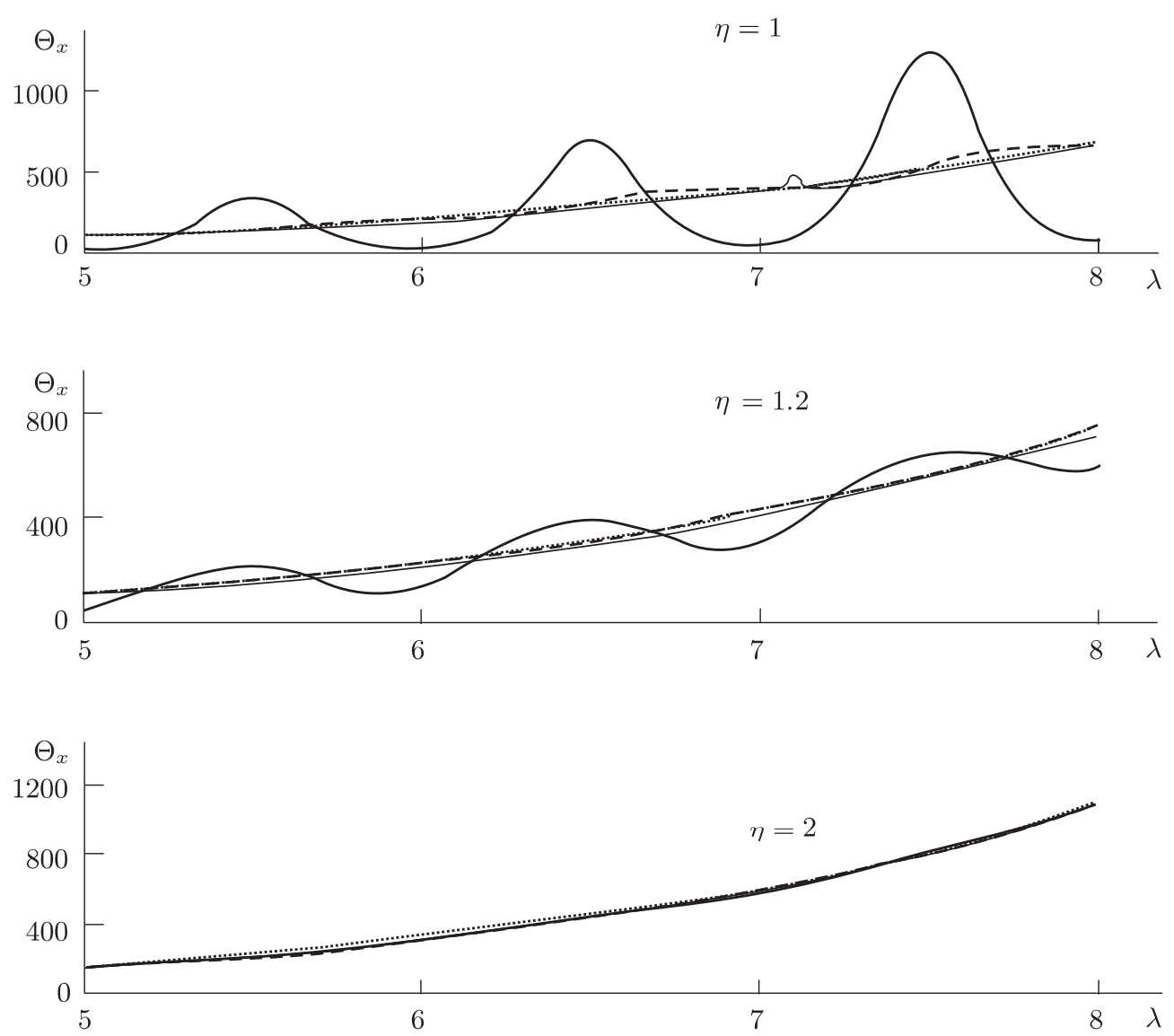

Рис. 1. Момент инерции (16) (сплошная линия), его усредненное по Струтинскому значение (тонкая сплошная линия), твердотельное значение (19) (штриховая линия) и его приближение Томаса-Ферми (20) (пунктирная линия) как функции химического потенциала $\lambda$ для некоторых значений параметра деформаций и температуры $T=0.1 \hbar \omega_{0}$. Все моменты инерции приведены в единицах $\hbar / \omega_{0}, \lambda$ - в единицах $\hbar \omega_{0}$.

На рис. 1, который взят из работы [13], представлены результаты численного расчета момента инерции (16) системы нуклонов в деформированном гармоническом потенциале в зависимости от химического потенциала для различных значений параметра деформаций $\eta$ при температуре $T=0.1 \hbar \omega_{0}$. Рис. 1 демонстрирует наличие осцилляций в зависимости момента инерции от химического потенциала, причем амплитуда осцилляций увеличивается при приближении деформации к сферическому пределу. На рисунке для сравнения также показана зависимость квантово-механического твердотельного момента инерции

$$
\Theta_{x}^{\mathrm{rig}}=2 m \sum_{\alpha} f\left(\varepsilon_{\alpha}-\lambda\right)\left\langle\alpha\left|y^{2}+z^{2}\right| \alpha\right\rangle=2 \hbar\left(\frac{N_{y}}{\omega_{\perp}}+\frac{N_{z}}{\omega_{z}}\right) .
$$


Из рис. 1 видно, что осцилляции $\Theta_{x}^{\text {rig }}(\lambda)$ выделяются всюду относительно приближения Томаса-Ферми

$$
\Theta_{x, \mathrm{TF}}^{\mathrm{rig}}=m \int \mathbf{d} \mathbf{r} r_{\perp x}^{2} \rho_{\mathrm{TF}}(\vec{r})=\frac{m}{3 \pi^{2} \hbar^{3}} \int \mathbf{d r}\left(y^{2}+z^{2}\right) p_{\mathrm{F}}^{2}(r)=\frac{1+\eta^{2}}{3 \omega_{\perp}^{2}} \frac{\lambda^{4}}{4 \eta^{2}\left(\hbar \omega_{0}\right)^{3}}
$$

где $p_{\mathrm{F}}(\mathbf{r})=\sqrt{2 m\left(\varepsilon_{\mathrm{F}}-m\left[\left(x^{2}+y^{2}\right) \omega_{\perp}^{2}+\omega_{z}^{2} z^{2}\right] / 2\right)}$, с томас-фермиевской плотностью состояний $\rho_{\mathrm{F}}(\mathbf{r})=p_{\mathrm{F}}^{3}(\mathbf{r}) / 3 \pi^{2} \hbar^{3}$.

Большие осцилляции полного момента инерции $\Theta_{x}$ по сравнению с осцилляциями $\Theta_{x}^{\text {rig }}$ при малых деформациях нетрудно объяснить исходя из выражения (16), которое удобно переписать в виде

$$
\Theta_{x}=\Theta_{x}^{\mathrm{rig}}+\frac{4 \eta^{2}}{\omega_{\perp}^{2}\left(\eta^{2}-1\right)} q
$$

где $q=\omega_{z} N_{z}-\omega_{\perp} N_{\perp}$. Соответственно оболочечная (осцилляционная) поправка будет равна

$$
\delta \Theta_{x}=\delta \Theta_{x}^{\mathrm{rig}}+\frac{4 \eta^{2}}{\omega_{\perp}^{2}\left(\eta^{2}-1\right)} \delta q
$$

т. е. она определяется оболочечными поправками $\delta \Theta_{x}^{\text {rig }}$ и $\delta q$. Функция $q$ характеризует нарушение условия статистически равновесного вращения

$$
\omega_{x} N_{x}=\omega_{y} N_{y}=\omega_{z} N_{z}
$$

и наличие даже небольших оболочечных поправок в ней за счет наличия множителя $\eta^{2}-1$ в знаменателе приводит при приближении к сферическому пределу к значительному увеличению амплитуды осцилляций.

Осцилляционное поведение момента инерции в некотором роде аналогично появлению осцилляций де Гааза-Ван Альфена в электронном газе, которые связаны с квантованием уровней Ландау в магнитном поле. Подобные осцилляции термодинамических характеристик проявляются во многих квантовых конечных ферми-системах (см., например, [18]) и являются следствием дискретности состояний, когда температура $T$ становится меньше или порядка расстояния между уровнями $\delta \sim E_{\mathrm{F}} / N\left(E_{\mathrm{F}}-\right.$ энергия ферми-системы, $N$ - число частиц в системе).

\section{3. ФОРМУЛИРОВКА МЕТОДА РАСЧЕТА МОМЕНТА ИНЕРЦИИ}

В данном разделе сформулируем общие положения математической методики расчета момента инерции (16) системы нуклонов в адиабатическом пределе, в рамках которой, на наш взгляд, наиболее удобно произвести разделение выражения (16) на гладкую и осциллирующую компоненты. Предлагаемая методика базируется на применении преобразования Меллина и, как будет показано ниже, в приложениях не ограничивается только расчетом выражения вида (16). Отметим, что ниже мы примем $\hbar=1, \omega_{0}=1$. 
Представим выражение для функции Ферми, входящей в выражения (18) для $N_{ \pm}$, в виде

$$
f(\varepsilon-\lambda)=\frac{1}{e^{(\varepsilon-\lambda) / T}+1}=\frac{1}{\kappa e^{\left(\varepsilon-\varepsilon_{0}\right) / T}+1} \equiv g(\kappa, \varepsilon),
$$

где

$$
\kappa=e^{\left(\varepsilon_{0}-\lambda\right) / T} .
$$

Продолжим область возможных значений химического потенциала $\lambda$ на интервал $\left(-\infty, \varepsilon_{0}\right)$ и будем считать далее, что $\lambda$ может принимать значения на всей действительной оси, т. е. $-\infty<\lambda<\infty$. Необходимость выполнения этого шага связана с допущением изменения величины $\kappa$ в $(25)$ в интервале $(0, \infty)$ для возможности осуществления преобразования Меллина в (24) по этому параметру. При этом, конечно, в окончательном выражении вклад от нефизической области значений $\lambda\left(-\infty, \varepsilon_{0}\right)$ будет исключен. Осуществляем преобразование Меллина функции $g(\kappa, \varepsilon)$ по аргументу $\kappa$ :

$$
\begin{aligned}
g(\kappa, \varepsilon) & \stackrel{\mathrm{MT}}{=} \widehat{G}(s, \varepsilon)=\int_{0}^{\infty} g(\kappa, \varepsilon) \kappa^{s-1} d \kappa= \\
& =\frac{\pi}{\sin (\pi s)} e^{-s\left(\varepsilon-\varepsilon_{0}\right) / T}, \quad 0<\gamma=\operatorname{Re} s<1 .
\end{aligned}
$$

Используя это выражение, произведем преобразование Меллина в формулах (18) для $N_{ \pm}$и получим следующие выражения для меллин-образов этих сумм:

$$
N_{ \pm} \stackrel{\mathrm{MT}}{=} \widehat{N}_{ \pm}(s)=\frac{\pi}{\sin (\pi s)} \sum_{n_{\perp}, n_{z}}\left(n_{\perp}+1\right)\left(n_{z}+\frac{1}{2} \pm \frac{1}{2}\left(n_{\perp}+1\right)\right) e^{-s \omega_{\perp}\left(n_{\perp}+n_{z} / \eta\right) / T} .
$$

Предполагая, что число квантов $n_{\perp}, n_{z}$ изменяется неограниченно, что существенно не скажется в дальнейших расчетах, поскольку в суммах для $N_{ \pm}$основной вклад дает область значений энергий вблизи энергии Ферми, проводим несложное суммирование в $(27)$ и окончательно находим следующие выражения для $\widehat{N}_{ \pm}(s)$ :

$$
\widehat{N}_{ \pm}(s)=\frac{\pi e^{s \varepsilon_{0} / T}}{16 \sin (\pi s)} \cdot \frac{\operatorname{sh}\left(s \omega_{\perp}(\eta \pm 1) / 2 \eta T\right)}{\operatorname{sh}^{3}\left(s \omega_{\perp} / 2 T\right) \operatorname{sh}^{2}\left(s \omega_{\perp} / 2 \eta T\right)} .
$$

Переход от меллин-образов (28) непосредственно к функциям $N_{ \pm}$осуществляется с помощью обратного преобразования Меллина по формуле

$$
N_{ \pm}=\frac{1}{2 \pi i} \int_{\gamma-i \infty}^{\gamma+i \infty} \widehat{N}_{ \pm}(s) \kappa^{-s} d s
$$

Для расчета интеграла Меллина-Барнса в правой части последней формулы необходимо прямую интегрирования дополнить до контура правой или левой половиной окружности (см. рис. 2), имея в виду, что интегралы от подынтегральной функции в (29) по половинкам окружностей равны нулю. Это нетрудно проверить прямыми вычислениями. Приведя интегралы (29) к контурным интегралам, мы можем воспользоваться интегральной теоремой Коши:

$$
\begin{aligned}
N_{ \pm} & =\frac{1}{2 \pi i} \int_{\gamma-i \infty}^{\gamma+i \infty} \widehat{N}_{ \pm}(s) \kappa^{-s} d s=\frac{1}{2 \pi i} \oint_{L_{ \pm}} \widehat{N}_{ \pm}(s) \kappa^{-s} d s= \\
& = \pm \sum_{k} \operatorname{Res}_{s_{k}}\left[\widehat{N}_{ \pm}(s) \kappa^{-s}\right]
\end{aligned}
$$




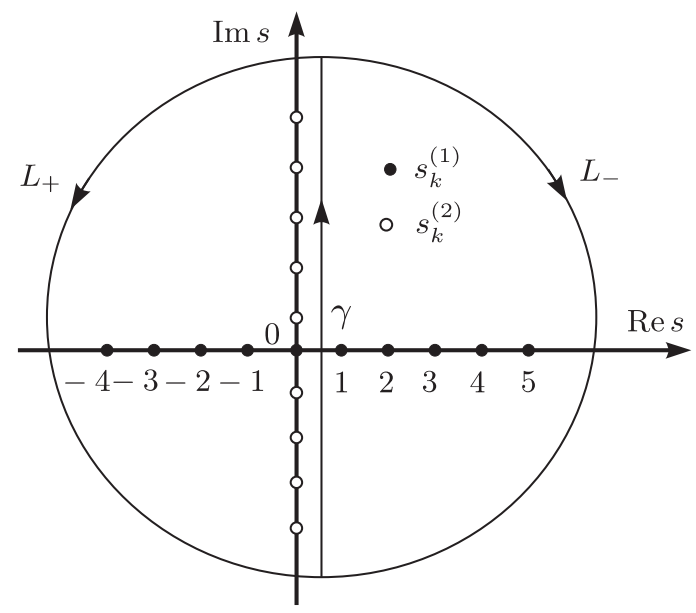

Рис. 2. Схематическое изображение положения полюсов функций (28) и контуров $L_{ \pm}$в комплексной плоскости.

где $L_{+}$и $L_{-}$определяют контуры с положительным и отрицательным обходом соответственно (см. рис. 2$), s_{k}$ - полюсы функций $\widehat{N}_{ \pm}(s) \kappa^{-s}$, попавшие в соответствующие контуры. Из анализа выражений $(28)$ для $\widehat{N}_{ \pm}(s)$ следует, что полюсы функций $\widehat{N}_{ \pm}(s) \kappa^{-s}$ можно разделить на две группы:

$$
s_{k}^{(1)}=k, \quad k=0, \pm 1, \pm 2, \ldots
$$

причем при $k \neq 0$ это простые полюсы функции $\pi / \sin (\pi s)$, а при $k=0$ имеем полюс пятого порядка, и

$$
s_{k}^{(2)}=\left\{\begin{array}{ll}
s_{k}^{\prime}=\frac{T}{\omega_{\perp}} 2 \pi k i & - \text { нули функции } \operatorname{sh}^{3}\left(s \omega_{\perp} / 2 T\right), \\
s_{k}^{\prime \prime}=\frac{\eta T}{\omega_{\perp}} 2 \pi k i & - \text { нули функции } \operatorname{sh}^{2}\left(s \omega_{\perp} / 2 \eta T\right),
\end{array} \quad k= \pm 1, \pm 2, \ldots\right.
$$

Такое разделение полюсов мы произвели по следующей причине. Полюсы первой группы $s_{k}^{(1)}$ располагаются на действительной оси (см. рис. 2) и, как нетрудно заметить, определяют гладкую составляющую $\bar{N}_{ \pm}$функций $N_{ \pm}$, а следовательно, и гладкую составляющую $\bar{\Theta}_{x}$ самого момента инерции (16). Напротив, полюсы второй группы являются чисто мнимыми и определяют осциллирующую составляющую $\widetilde{N}_{ \pm}$функций $N_{ \pm}$, а следовательно, и осциллирующую составляющую $\widetilde{\Theta}_{x}$ момента инерции (16). В результате имеем

$$
\begin{aligned}
& N_{ \pm}=\bar{N}_{ \pm}+\widetilde{N}_{ \pm}, \quad \bar{N}_{ \pm}= \pm \sum_{k} \underset{s_{k}^{(1)}}{\operatorname{Res}}\left[\widehat{N}_{ \pm}(s) \kappa^{-s}\right] \\
& \widetilde{N}_{ \pm}= \pm \sum_{k} \operatorname{Res}_{s_{k}^{(2)}}\left[\widehat{N}_{ \pm}(s) \kappa^{-s}\right] \Rightarrow \Theta_{x}=\bar{\Theta}_{x}+\widetilde{\Theta}_{x}
\end{aligned}
$$

Таким образом, посредством преобразования Меллина мы смогли разделить зависимость момента инерции $\Theta_{x}(\lambda)$ на гладкую и осциллирующую составляющие, 
вклады в которые дают действительные и с отличной от нуля мнимой частью полюсы меллин-образов соответственно. Отметим, что, как уже упоминалось выше, данная процедура разделения функции на гладкую и осциллирующую составляющие не ограничивается рассмотрением только решаемой здесь задачи, но может быть применена и к другим подобным задачам, например к задаче работы [18], если возникает потребность в явных аналитических выражениях указанных вкладов.

Возвращаясь к рассматриваемой здесь задаче, отметим, что полюсы $s_{k}^{\prime}$ и $s_{k}^{\prime \prime}$ второй группы не пересекаются и являются соответственно полюсами третьего и второго порядков в случае, если параметр деформации $\eta$ принимает иррациональные значения (несоизмеримые частоты). В случае рациональных значений (соизмеримые частоты) параметра $\eta=m / n, m, n \in \mathbb{N}$, имеется пересечение этих полюсов: $s_{k_{1}}^{\prime}=s_{k_{2}}^{\prime \prime}$ при $k_{1} / k_{2}=m / n$. Поэтому при расчете осциллирующих составляющих необходимо разделять эти два случая. Также следует пояснить вопрос о выборе контуров в (30). Выбор левого $L_{+}$или правого $L_{-}$контуров приведет, как мы покажем ниже, к выражениям, которые справедливы для различных областей значений $\lambda$ и являются аналитическими продолжениями по отношению друг к другу. Именно здесь и происходит исключение вклада в момент инерции от нефизических значений химического потенциала $\lambda$.

\section{4. РАСЧЕТ ГЛАДКОЙ СОСТАВЛЯЮЩЕЙ МОМЕНТА ИНЕРЦИИ}

Гладкая составляющая функций $N_{ \pm}$, как было отмечено выше, определяется вычетами в полюсах, которые расположены на действительной оси. Вычислим вычеты функций $\widehat{N}_{ \pm}(s) \kappa^{-s}$ в этих точках. Прежде чем производить непосредственные вычисления вычетов, примем следующие удобные в дальнейшем обозначения:

$$
z=\frac{s \omega_{\perp}}{T}, \quad \phi=\frac{\lambda}{\omega_{\perp}}, \quad \tau=\frac{T}{\omega_{\perp}},
$$

тогда гладкие составляющие $\bar{N}_{ \pm}$при выборе сначала контура $L_{+}$, как это следует из (33) и рис. 2 , будут вычисляться по формулам

$$
\begin{aligned}
\bar{N}_{ \pm} & =\operatorname{Res}_{z=0}\left[R_{ \pm}(z)\right]+\sum_{k=1}^{\infty} \operatorname{Res}_{z=-k / \tau}\left[R_{ \pm}(z)\right], \\
R_{ \pm}(z) & =\frac{\pi \tau e^{\phi z}}{16 \sin (\pi z \tau)} \frac{\operatorname{sh}(z(\eta \pm 1) / 2 \eta)}{\operatorname{sh}^{3}(z / 2) \operatorname{sh}^{2}(z / 2 \eta)} .
\end{aligned}
$$

Несложные, но достаточно громоздкие вычисления ( $z=0$ - полюс пятого порядка) приводят к следующим выражениям для вычетов, входящих в (35):

$$
\begin{aligned}
& \operatorname{Res}_{z=0}\left[R_{ \pm}(z)\right]=\frac{\eta \pm 1}{5760 \eta^{3}}\left\{7 \mp 28 \eta+28 \eta^{2} \mp 48 \eta^{3}+24 \eta^{4}+\right. \\
& +120 \eta^{2}\left( \pm 2 \eta-1-2 \eta^{2}\right) \phi^{2}+240 \eta^{4} \phi^{4}+ \\
& \left.+40 \pi^{2} \eta^{2}\left(-1 \pm 2 \eta-2 \eta^{2}+12 \eta^{2} \phi^{2}\right) \tau^{2}+112 \pi^{4} \eta^{4} \tau^{4}\right\}, \\
& \underset{z=-k / \tau}{\operatorname{Res}}\left[R_{ \pm}(z)\right]=\frac{(-1)^{k} e^{-k \phi / \tau} \operatorname{sh}(k(\eta \pm 1) / 2 \eta \tau)}{16 \operatorname{sh}^{3}(k / 2 \tau) \operatorname{sh}^{2}(k / 2 \eta \tau)} \text {. }
\end{aligned}
$$


Подстановка полученных выражений в (35) и последующая подстановка результата в формулу для момента инерции (16) приводят к следующему выражению для гладкой составляющей момента инерции:

$$
\begin{aligned}
\bar{\Theta}_{x}= & \frac{1}{\omega_{\perp}\left(\eta^{2}-1\right)}\left\{(\eta-1)^{3} \bar{N}_{+}+(\eta+1)^{3} \bar{N}_{-}\right\}= \\
= & \frac{1}{2880 \omega_{\perp} \eta^{3}}\left\{7+91 \eta^{2}+148 \eta^{4}+24 \eta^{6}-40 \eta^{2}\left(1+7 \eta^{2}+2 \eta^{4}\right)\left(3 \phi^{2}+\pi^{2} \tau^{2}\right)+\right. \\
& \left.+16 \eta^{4}\left(1+\eta^{2}\right)\left(15 \phi^{4}+30 \pi^{2} \phi^{2} \tau^{2}+7 \pi^{4} \tau^{4}\right)\right\}+\frac{1}{16 \omega_{\perp}\left(\eta^{2}-1\right)} \times \\
& \times \sum_{k=1}^{\infty} \frac{(-1)^{k} e^{-k \phi / \tau}\left\{(\eta-1)^{3} \operatorname{sh}(k(\eta+1) / 2 \eta \tau)+(\eta+1)^{3} \operatorname{sh}(k(\eta-1) / 2 \eta \tau)\right\}}{\operatorname{sh}^{3}(k / 2 \tau) \operatorname{sh}^{2}(k / 2 \eta \tau)} .
\end{aligned}
$$

При выборе правого контура $L_{-}$и полюсов, попавших в него (см. рис. 2), получим другое выражение для гладкой составляющей момента инерции:

$$
\begin{aligned}
\bar{\Theta}_{x}= & -\frac{1}{\omega_{\perp}\left(\eta^{2}-1\right)} \sum_{k=1}^{\infty}\left\{(\eta-1)^{3} \operatorname{Res}_{z=k / \tau}\left[R_{+}(z)\right]+(\eta+1)^{3} \underset{z=k / \tau}{\operatorname{Res}}\left[R_{-}(z)\right]\right\}= \\
= & \frac{1}{16 \omega_{\perp}\left(\eta^{2}-1\right)} \times \\
& \times \sum_{k=1}^{\infty} \frac{(-1)^{k+1} e^{k \phi / \tau}\left\{(\eta-1)^{3} \operatorname{sh}(k(\eta+1) / 2 \eta \tau)+(\eta+1)^{3} \operatorname{sh}(k(\eta-1) / 2 \eta \tau)\right\}}{\operatorname{sh}^{3}(k / 2 \tau) \operatorname{sh}^{2}(k / 2 \eta \tau)} .
\end{aligned}
$$

Исследование на сходимость ряда в (39) показывает, что этот ряд сходится в области $-\infty<\lambda<\varepsilon_{0}$, которая, как упоминалось выше, является нефизической. Таким образом, выражение (39) для гладкой составляющей момента инерции не принимается в рассмотрение, а выражение (38), которое, очевидно, будет аналитическим продолжением выражения (39) для $\bar{\Theta}_{x}$ в область $\varepsilon_{0} \leqslant \lambda<\infty$, выбирается в качестве окончательного выражения для искомой гладкой составляющей момента инерции.

При $\tau=0$ выражение для гладкой составляющей момента инерции принимает вид

$$
\begin{aligned}
\left.\bar{\Theta}_{x}(\tau)\right|_{\tau=0}= & \frac{1}{2880 \omega_{\perp} \eta^{3}}\left\{7+91 \eta^{2}+148 \eta^{4}+24 \eta^{6}-\right. \\
& \left.-120 \eta^{2}\left(1+7 \eta^{2}+2 \eta^{4}\right) \phi^{2}+240 \eta^{4}\left(1+\eta^{2}\right) \phi^{4}\right\},
\end{aligned}
$$

который, как нетрудно заметить, отличается от выражения для твердотельного момента инерции в приближении Томаса-Ферми (20). Формула (40) приближается к выражению (20) при достаточно высоких значениях $\lambda$. При низких температурах и вблизи сферического предела $(\eta=1)$ можно записать следующую аппроксимацию выражения (38):

$$
\bar{\Theta}_{x} \approx \frac{80\left(\eta^{2}+5\right) \lambda^{4}-120\left(\eta^{2}+9\right) \lambda^{2}+9\left(\eta^{2}+29\right)}{2880}+\frac{\pi^{2}}{38}\left(12 \lambda^{2}-5\right) T^{2} .
$$




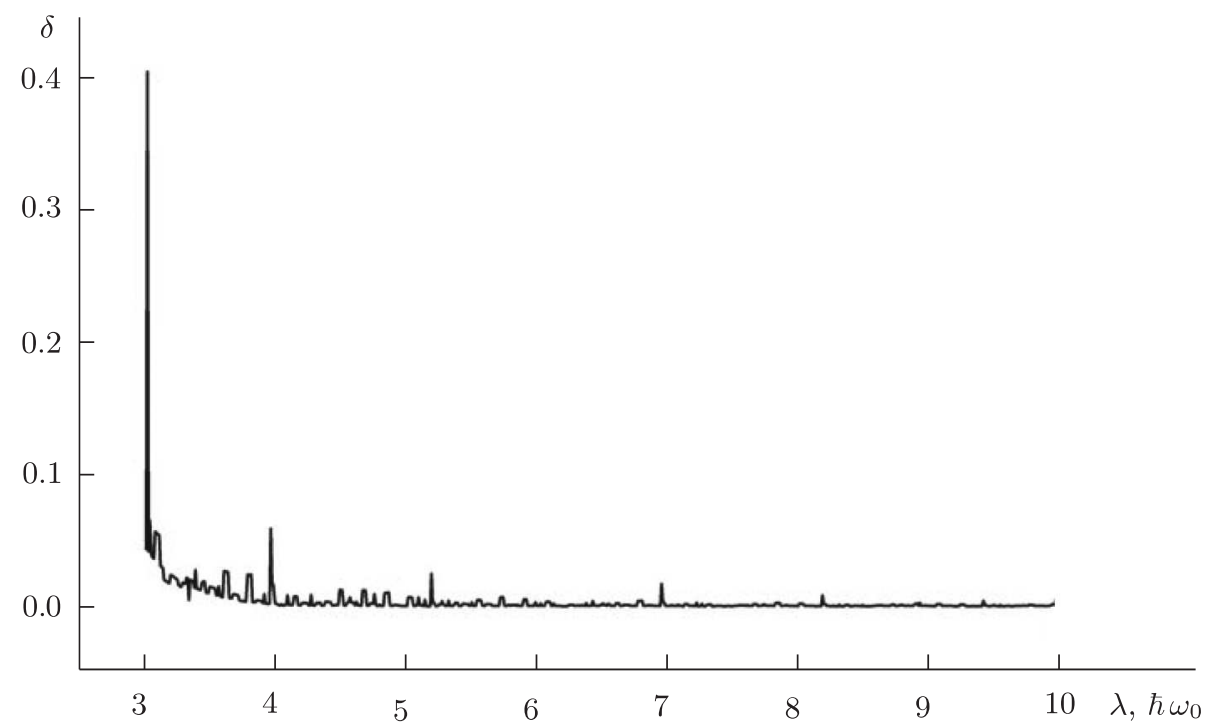

Рис. 3. Относительное отклонение $\delta=\left|\bar{\Theta}_{x}-\Theta_{x, \text { av }}\right| \cdot 100 \% / \Theta_{x, \text { av }}$ в процентах гладкой составляющей момента инерции $\bar{\Theta}_{x}$ (38) от момента инерции, сглаженного по Струтинскому $\Theta_{x, \text { av }}$, как функция химического потенциала $\lambda$ для параметра деформации $\eta=6 / 5$ и температуры $T=0.1 \hbar \omega_{0}$.

Далее продемонстрируем сравнение выражения для гладкой составляющей момента инерции (38), полученное в рамках предлагаемого метода, с аналогичным выражением, которое следует из метода сглаживания Струтинского [9], [10]. Согласно процедуре, предложенной Струтинским, для получения сглаженного выражения для момента инерции в точной формуле для момента инерции (16) следует заменить функции $N_{ \pm}$на их сглаженные аналоги $N_{\mathrm{av} \pm}$, которые определяются по формулам

$$
N_{\mathrm{av} \pm}=\sum_{n_{\perp}, n_{z}} \tilde{f}\left(\varepsilon_{n_{\perp} n_{z}}-\lambda\right)\left(n_{\perp}+1\right)\left(n_{z}+\frac{1}{2} \pm \frac{n_{\perp}+1}{2}\right)
$$

где

$$
\tilde{f}(\varepsilon-\lambda)=\frac{1}{\gamma} \int_{-\infty}^{\infty} d \lambda^{\prime} f\left(\varepsilon-\lambda^{\prime}\right) \xi_{M}\left(\frac{\lambda^{\prime}-\lambda}{\gamma}\right), \quad \xi_{M}(x)=\frac{1}{\sqrt{\pi}} e^{-x^{2}} P_{2 M}(x) .
$$

Здесь $P_{2 M}(x)=\sum_{k=0,2, \ldots}^{2 M} \alpha_{k} H_{k}(x)$, где $H_{k}(x)$ - полиномы Эрмита, $\alpha_{k}=-\alpha_{k-2} / 2 k$ $\left(\alpha_{0}=1\right)$, определяет корректирующий полином порядка $2 M$ (обычно $M=4-10$ ). Ширина кривой Гаусса $\gamma$ и порядок полинома $M$ выбираются на плато зависимости оболочечной поправки момента инерции $\delta \Theta_{x}(\varepsilon)=\Theta_{x}(\varepsilon)-\Theta_{x, \text { av }}(\varepsilon)$ от $\gamma$ для нескольких значений $M$ при фиксированном $\varepsilon$. Вычисления показывают, что $\gamma=1.5-2.5 \omega_{0}$ при $M=4-8$. Отметим, что процедура выделения гладкой составляющей по Струтинскому является численной процедурой, поэтому развиваемый в работе метод выделения гладкой составляющей обладает некоторым преимуществом, поскольку позволяет получить аналитические выражения. 
Сравнение значений гладкой составляющей момента инерции, полученных по формуле (38), с соответствующими значениями, вычисленными по процедуре сглаживания Струтинского, показывает хорошее согласие. Для наглядной демонстрации этого вывода на рис. 3 изображена зависимость от $\lambda$ относительного отклонения $\delta=\left|\bar{\Theta}_{x}-\Theta_{x, \text { av }}\right| \cdot 100 \% / \Theta_{x, \text { av }}$ в процентах момента инерции $\bar{\Theta}_{x}(38)$ от сглаженного по Струтинскому момента инерции $\Theta_{x, \text { av }}$ для параметра деформации $\eta=6 / 5$ и температуры $T=0.1 \hbar \omega_{0}$. Из рисунка видно, что совпадение гладких составляющих обоих методов прекрасное, а отклонения заметны лишь при малых значениях $\lambda$. Таким образом, на основании численных расчетов можно сделать вывод об эквивалентности предлагаемого метода выделения гладкой составляющей момента инерции методу сглаживания Струтинского.

\section{5. РАСЧЕТ ОСЦИЛЛИРУЮЩЕЙ СОСТАВЛЯЮЩЕЙ МОМЕНТА ИНЕРЦИИ}

В данном разделе мы проведем расчет осциллирующей составляющей момента инерции, следуя общей методике, изложенной в разделе 3 . Как уже упоминалось в разделе 3, при расчете рассмотрим два случая: иррациональных и рациональных значений параметра деформации $\eta$.

5.1. Случай иррациональных значений $\eta$. В случае иррациональных значений параметра $\eta$ полюсы второй группы $s_{k}^{\prime}$ и $s_{k}^{\prime \prime}$, которые находятся внутри контура $L_{+}$(см. рис. 2), не пересекаются, поэтому согласно (33) для осциллирующих составляющих функций $\tilde{N}_{ \pm}$при выборе контура $L_{+}$имеем

$$
\begin{aligned}
\tilde{N}_{ \pm}=\sum_{k} \operatorname{Res}_{z_{k}^{\prime}}\left[R_{ \pm}(z)\right]+\sum_{k} \operatorname{Res}_{z_{k}^{\prime \prime}}\left[R_{ \pm}(z)\right]=\sum_{k=1}^{\infty}\left(\operatorname{Res}_{z=2 \pi i k}\left[R_{ \pm}(z)\right]+\operatorname{Res}_{z=-2 \pi i k}\left[R_{ \pm}(z)\right]\right)+ \\
+\sum_{k=1}^{\infty}\left(\underset{z=2 \pi i k \eta}{\operatorname{Res}}\left[R_{ \pm}(z)\right]+\underset{z=-2 \pi i k \eta}{\operatorname{Res}}\left[R_{ \pm}(z)\right]\right)
\end{aligned}
$$

где $z_{k}^{\prime}=s_{k}^{\prime} / \tau, z_{k}^{\prime \prime}=s_{k}^{\prime \prime} / \tau$, а $R_{ \pm}(z)$ определяются в (35). Снова, не приводя деталей расчета, которые являются элементарными, но громоздкими, запишем сразу результаты вычислений вычетов, входящих в (42):

$$
\begin{aligned}
\operatorname{Res}_{z=2 \pi i k}\left[R_{ \pm}(z)\right]+\underset{z=-2 \pi i k}{\operatorname{Res}_{ \pm}}\left[R_{ \pm}(z)\right]=A_{k}^{ \pm} \cos (2 \pi k \phi)+B_{k}^{ \pm} \sin (2 \pi k \phi) & \\
A_{k}^{ \pm}= \pm & \frac{\pi \tau}{8 \eta^{2} \sin (\pi k / \eta) \operatorname{sh}\left(2 \pi^{2} k \tau\right)}\left\{(1 \mp 2 \eta)\left(1+2 \operatorname{ctg}^{2}\left(\frac{\pi k}{\eta}\right)\right)-4 \eta^{2}\left(\phi^{2}+\pi^{2} \tau^{2}\right)+\right. \\
& \left.+4 \pi \eta \tau \operatorname{cth}\left(2 \pi^{2} k \tau\right)\left[(1 \mp \eta) \operatorname{ctg} \frac{\pi k}{\eta}+2 \pi \eta \tau \operatorname{cth}\left(2 \pi^{2} k \tau\right)\right]\right\} \\
B_{k}^{ \pm}= & \pm \frac{\pi \tau \phi}{2 \eta \sin (\pi k / \eta) \operatorname{sh}\left(2 \pi^{2} k \tau\right)}\left\{(1 \mp \eta) \operatorname{ctg} \frac{\pi k}{\eta}+2 \pi \eta \tau \operatorname{cth}\left(2 \pi^{2} k \tau\right)\right\} \\
z=2 \pi i k \eta & \operatorname{Res}\left[R_{ \pm}(z)\right]+\underset{z=-2 \pi i k \eta}{\operatorname{Res}}\left[R_{ \pm}(z)\right]=C_{k}^{ \pm} \cos (2 \pi k \eta \phi)+D_{k}^{ \pm} \sin (2 \pi k \eta \phi) \\
C_{k}^{ \pm}= & \frac{\pi \tau \eta(-1)^{k+1}}{4 \sin ^{2}(\pi k \eta) \operatorname{sh}\left(2 \pi^{2} k \eta \tau\right)}\left\{(2 \eta \mp 1) \operatorname{ctg}(\pi k \eta)+2 \pi \eta \tau \operatorname{cth}\left(2 \pi^{2} k \eta \tau\right)\right\} \\
D_{k}^{ \pm}= & \frac{(-1)^{k+1} \pi \tau \eta^{2} \phi}{2 \sin ^{2}(\pi k \eta) \operatorname{sh}\left(2 \pi^{2} k \eta \tau\right)}
\end{aligned}
$$


Подстановка полученных выражений в (42) приводит к следующему результату для $\widetilde{N}_{ \pm}$:

$$
\widetilde{N}_{ \pm}=\sum_{k=1}^{\infty}\left\{A_{k}^{ \pm} \cos (2 \pi k \phi)+B_{k}^{ \pm} \sin (2 \pi k \phi)+C_{k}^{ \pm} \cos (2 \pi k \eta \phi)+D_{k}^{ \pm} \sin (2 \pi k \eta \phi)\right\} .
$$

Зная осциллирующие составляющие функций $N_{ \pm}$, по формуле (16) восстанавливаем осциллирующую составляющую момента инерции:

$$
\begin{aligned}
& \widetilde{\Theta}_{x}=\sum_{k=1}^{\infty}\left(A_{k} \cos (2 \pi k \phi)+B_{k} \sin (2 \pi k \phi)+C_{k} \cos (2 \pi k \eta \phi)+D_{k} \sin (2 \pi k \eta \phi)\right), \\
& A_{k}=\frac{(\eta-1)^{3} A_{k}^{+}+(\eta+1)^{3} A_{k}^{-}}{\omega_{\perp}\left(\eta^{2}-1\right)}= \\
& =-\frac{\pi \tau}{4 \omega_{\perp} \eta^{2}\left(\eta^{2}-1\right) \sin (\pi k / \eta) \operatorname{sh}\left(2 \pi^{2} k \tau\right)} \times \\
& \times\left\{1-\eta^{2}\left(-9+4 \pi^{2} \tau^{2}\left(3 \eta^{2}+1\right)+4 \phi^{2}+2 \eta^{2}\left(6 \phi^{2}-1\right)\right)+\right. \\
& +2\left(1+9 \eta^{2}+2 \eta^{4}\right) \operatorname{ctg}^{2} \frac{\pi k}{\eta}+4 \pi \tau \operatorname{cth}\left(2 \pi^{2} k \tau\right) \times \\
& \left.\times\left[\eta\left(1+6 \eta^{2}+\eta^{4}\right) \operatorname{ctg} \frac{\pi k}{\eta}+2 \pi \eta^{2} \tau\left(3 \eta^{2}+1\right) \operatorname{cth}\left(2 \pi^{2} k \tau\right)\right]\right\}, \\
& B_{k}=\frac{(\eta-1)^{3} B_{k}^{+}+(\eta+1)^{3} B_{k}^{-}}{\omega_{\perp}\left(\eta^{2}-1\right)}= \\
& =-\frac{\pi \tau \phi\left[\left(1+6 \eta^{2}+\eta^{4}\right) \operatorname{ctg}(\pi k / \eta)+2 \pi \eta \tau\left(3 \eta^{2}+1\right) \operatorname{cth}\left(2 \pi^{2} k \tau\right)\right]}{\omega_{\perp} \eta\left(\eta^{2}-1\right) \sin (\pi k / \eta) \operatorname{sh}\left(2 \pi^{2} k \tau\right)}, \\
& C_{k}=\frac{(\eta-1)^{3} C_{k}^{+}+(\eta+1)^{3} C_{k}^{-}}{\omega_{\perp}\left(\eta^{2}-1\right)}= \\
& =\frac{\pi \tau \eta(-1)^{k+1}\left[\left(2 \eta^{4}+9 \eta^{2}+1\right) \operatorname{ctg}(\pi k \eta)+2 \pi \eta^{2} \tau\left(3+\eta^{2}\right) \operatorname{cth}\left(2 \pi^{2} k \eta \tau\right)\right]}{2 \omega_{\perp}\left(\eta^{2}-1\right) \sin ^{2}(\pi k \eta) \operatorname{sh}\left(2 \pi^{2} k \eta \tau\right)}, \\
& D_{k}=\frac{(\eta-1)^{3} D_{k}^{+}+(\eta+1)^{3} D_{k}^{-}}{\omega_{\perp}\left(\eta^{2}-1\right)}=\frac{(-1)^{k+1} \pi \tau \eta^{3}\left(\eta^{2}+3\right) \phi}{\omega_{\perp}\left(\eta^{2}-1\right) \sin ^{2}(\pi k \eta) \operatorname{sh}\left(2 \pi^{2} k \eta \tau\right)} .
\end{aligned}
$$

Из анализа полученного выражения можно сделать следующие важные выводы. В осциллирующую составляющую $\widetilde{\Theta}_{x}(\lambda)$ момента инерции дают вклад два "периодических" процесса: процесс с "периодом", равным $\omega_{\perp}=\eta^{1 / 3}$, и процесс с "периодом", равным $\omega_{\perp} / \eta \equiv \omega_{z}=\eta^{-2 / 3}$. Анализ выражений для коэффициентов $A_{k}, B_{k}$, $C_{k}, D_{k}(45)$ показывает, что осцилляции момента инерции затухают с ростом температуры по экспоненциальному закону, $\left\{A_{k}, B_{k}\right\} \sim e^{-2 \pi^{2} k \tau},\left\{C_{k}, D_{k}\right\} \sim e^{-2 \pi^{2} k \eta \tau}$, и усиливаются при приближении к сферическому пределу $(\eta \rightarrow 1)$. 
Вблизи сферического предела коэффициенты $A_{k}, B_{k}, C_{k}, D_{k}$ можно аппроксимировать выражениями

$$
\begin{array}{ll}
A_{k} \approx-\frac{6 \pi T}{\left(\eta^{2}-1\right) \sin ^{3}(\pi k / \eta) \operatorname{sh}\left(2 \pi^{2} k T\right)}, & B_{k} \approx \frac{8(-1)^{k+1} \pi T \lambda}{\left(\eta^{2}-1\right) \sin ^{2}(\pi k / \eta) \operatorname{sh}\left(2 \pi^{2} k T\right)}, \\
C_{k} \approx-\frac{6 \pi T}{\left(\eta^{2}-1\right) \sin ^{3}(\pi k \eta) \operatorname{sh}\left(2 \pi^{2} k T\right)}, & D_{k} \approx \frac{4(-1)^{k+1} \pi T \lambda}{\left(\eta^{2}-1\right) \sin ^{2}(\pi k \eta) \operatorname{sh}\left(2 \pi^{2} k T\right)} .
\end{array}
$$

Из полученных формул видно, что коэффициенты $A_{k}, B_{k}, C_{k}, D_{k}$ при приближении к сферическому пределу растут. Однако неограниченного увеличения вклада осциллирующей составляющей момента инерции не происходит и в сферическом пределе она принимает следующее предельное выражение для высоких значений $\lambda$ :

$$
\left.\widetilde{\Theta}_{x}\right|_{\eta=1} \approx \sum_{k=1}^{\infty} \frac{(-1)^{k} 2 \pi^{2} k T \lambda^{4}}{3 \operatorname{sh}\left(2 \pi^{2} k T\right)}\left[\cos (2 \pi k \lambda)-\frac{8 \pi^{3} k T^{2}}{3 \lambda} \sin (2 \pi k \lambda)\right]
$$

В пределе $\tau=0$ осцилляции исчезают, и осциллирующая составляющая становится равной $\left.\widetilde{\Theta}_{x}\right|_{\tau=0, \eta=1}=-\left.\bar{\Theta}_{x}\right|_{\tau=0, \eta=1}$. В результате при $\tau=0$ в сферическом пределе момент инерции $\left.\Theta_{x}\right|_{\tau=0, \eta=1}=\left.\bar{\Theta}_{x}\right|_{\tau=0, \eta=1}+\left.\widetilde{\Theta}_{x}\right|_{\tau=0, \eta=1}=0$. Таким образом, сферически-симметричные ядра при нулевой температуре в рамках рассматриваемой модели не обладают моментом инерции. Отметим, что этот же результат предсказывает гидродинамическая модель, когда вращение ядра представляется как потенциальное движение жидкости во вращающейся оболочке.

5.2. Случай рациональных значений $\eta$. Как уже упоминалось в разделе 3 , в случае рациональных значений параметра деформации $\eta=m / n, m, n \in \mathbb{N}$, имеется пересечение полюсов второй группы: $s_{k_{1}}^{\prime}=s_{k_{2}}^{\prime \prime}$ при $k_{1} / k_{2}=m / n$. Поэтому необходимо пересчитать значения вычетов функций $R_{ \pm}(z)(35)$ в точках совпадения полюсов, поскольку теперь они становятся полюсами четвертого порядка. Для вычисления вычетов в полюсах $s_{k_{1}}^{\prime}=s_{k_{2}}^{\prime \prime}$ выбираем $k_{1}=m k, k_{2}=n k, k= \pm 1, \pm 2, \ldots$, и после несложных вычислений находим

$$
\begin{aligned}
\operatorname{Res}_{z=2 \pi i m k}\left[R_{ \pm}(z)\right]+\operatorname{Res}_{z=-2 \pi i m k}\left[R_{ \pm}(z)\right]=P_{k}^{ \pm} \cos (2 \pi m k \phi)+Q_{k}^{ \pm} \sin (2 \pi m k \phi), \\
P_{k}^{ \pm}=\frac{(-1)^{n k+1} \pi^{2} \tau^{2}(\eta \pm 1) \operatorname{cth}\left(2 \pi^{2} m k \tau\right)}{12 \eta \operatorname{sh}\left(2 \pi^{2} m k \tau\right)} \times \\
\quad \times\left[1+2 \eta\left(\mp 1-\eta\left(6 \phi^{2}+10 \pi^{2} \tau^{2}-1\right)\right)+24 \pi^{2} \tau^{2} \eta^{2} \operatorname{cth}^{2}\left(2 \pi^{2} m k \tau\right)\right], \\
Q_{k}^{ \pm}=\frac{(-1)^{n k+1} \pi \tau(\eta \pm 1) \phi}{12 \eta \operatorname{sh}\left(2 \pi^{2} m k \tau\right)} \times \\
\quad \times\left[1+2 \eta\left(\mp 1-\eta\left(2 \phi^{2}+6 \pi^{2} \tau^{2}-1\right)\right)+24 \pi^{2} \tau^{2} \eta^{2} \operatorname{cth}^{2}\left(2 \pi^{2} m k \tau\right)\right] .
\end{aligned}
$$


Подстановка полученных выражений в формулы для $\widetilde{N}_{ \pm}$и последующая подстановка результата в выражение для $\widetilde{\Theta}_{x}$ приводят к следующему выражению для осциллирующей составляющей момента инерции в случае рациональных значений $\eta$ :

$$
\begin{aligned}
\widetilde{\Theta}_{x}= & \sum_{k=1}^{\infty}\left(A_{k}^{\prime} \cos (2 \pi k \phi)+B_{k}^{\prime} \sin (2 \pi k \phi)+C_{k}^{\prime} \cos (2 \pi k \eta \phi)+D_{k}^{\prime} \sin (2 \pi k \eta \phi)\right), \\
A_{k}^{\prime}= & \begin{array}{ll}
A_{k}, & k / m \notin \mathbb{N}, \\
P_{k}, & k / m \in \mathbb{N},
\end{array} \quad B_{k}^{\prime}= \begin{cases}B_{k}, & k / m \notin \mathbb{N}, \\
Q_{k}, & k / m \in \mathbb{N},\end{cases} \\
C_{k}^{\prime}= & \left\{\begin{array}{ll}
C_{k}, & k / n \notin \mathbb{N}, \\
0, & k / n \in \mathbb{N},
\end{array} \quad D_{k}^{\prime}= \begin{cases}D_{k}, & k / n \notin \mathbb{N}, \\
0, & k / n \in \mathbb{N},\end{cases} \right. \\
P_{k}= & \frac{(-1)^{k+1} \pi^{2} \tau^{2} \operatorname{cth}\left(2 \pi^{2} k \tau\right)}{6 \eta \omega_{\perp} \operatorname{sh}^{2}\left(2 \pi^{2} k \tau\right)}\left[1+\eta^{2}\left(7+2 \eta^{2}-4\left(\eta^{2}+1\right)\left(3 \phi^{2}+5 \pi^{2} \tau^{2}\right)\right)+\right. \\
& \left.+24 \pi^{2} \tau^{2} \eta^{2}\left(\eta^{2}+1\right) \operatorname{cth}^{2}\left(2 \pi^{2} k \tau\right)\right], \\
Q_{k}= & \frac{(-1)^{k+1} \pi \tau \phi}{6 \eta \omega_{\perp} \operatorname{sh}^{2}\left(2 \pi^{2} k \tau\right)}\left[1+\eta^{2}\left(7+2 \eta^{2}-4\left(\eta^{2}+1\right)\left(\phi^{2}+3 \pi^{2} \tau^{2}\right)\right)+\right. \\
& \left.+24 \pi^{2} \tau^{2} \eta^{2}\left(\eta^{2}+1\right) \operatorname{cth}^{2}\left(2 \pi^{2} k \tau\right)\right] .
\end{aligned}
$$

В пределе $\tau \rightarrow 0$ коэффициенты $P_{k}, Q_{k}$ стремятся к предельным значениям

$$
\begin{aligned}
P_{k}^{0} & =(-1)^{k+1} \frac{6 \eta^{2}\left(1+\eta^{2}\right)+\pi^{2} k^{2}\left(1+7 \eta^{2}+2 \eta^{4}-12 \eta^{2}\left(1+\eta^{2}\right) \phi^{2}\right)}{24 \pi^{4} k^{4} \eta \omega_{\perp}} \\
Q_{k}^{0} & =(-1)^{k+1} \phi \frac{6 \eta^{2}\left(1+\eta^{2}\right)+\pi^{2} k^{2}\left(1+7 \eta^{2}+2 \eta^{4}-4 \eta^{2}\left(1+\eta^{2}\right) \phi^{2}\right)}{12 \pi^{3} k^{3} \eta \omega_{\perp}}
\end{aligned}
$$

Как нетрудно заметить из (49) и (50), гармоники, соответствующие совпадающим частотам, вследствие рациональности $\eta$ не усиливаются при приближении к сферическому пределу.

\section{6. ТВЕРДОТЕЛЬНЫЙ МОМЕНТ ИНЕРЦИИ}

Обсудим поведение момента инерции с изменением химического потенциала при выполнении условия статистически равновесного вращения (23), когда его выражение (16) переходит в твердотельное выражение (19). Чтобы воспользоваться результатами предыдущих разделов, представим выражение для твердотельного момента инерции (19) в виде

$$
\Theta_{x}^{\mathrm{rig}}=\frac{1}{\omega_{\perp}}\left((\eta+1) N_{+}+(\eta-1) N_{-}\right)
$$

где $N_{ \pm}$определяются в (33). Поскольку, как было показано в разделе 5 , функции $N_{ \pm}$состоят из гладкой и осциллирующей составляющих, то очевидно, что 
твердотельный момент инерции также будет содержать осциллирующую составляющую. Интересно оценить масштаб этих осцилляций и сравнить степень их проявления с осцилляциями момента инерции, когда статистически равновесное вращение нарушается.

Используя результаты раздела 5 для $\bar{N}_{ \pm}, \widetilde{N}_{ \pm}$и проводя аналогичные вычисления, для $\Theta_{x}^{\text {rig }}$ получим

$$
\Theta_{x}^{\mathrm{rig}}=\bar{\Theta}_{x}^{\mathrm{rig}}+\widetilde{\Theta}_{x}^{\mathrm{rig}}
$$

где гладкая составляющая $\bar{\Theta}_{x}^{\text {rig }}$ имеет вид

$$
\begin{aligned}
& \bar{\Theta}_{x}^{\mathrm{rig}}=\frac{1}{2880 \omega_{\perp} \eta^{3}}\left\{7-65 \eta^{2}+24 \eta^{4}-40 \eta^{2}\left(2 \eta^{4}+1-\eta^{2}\right)\left(3 \phi^{2}+\pi^{2} \tau^{2}\right)+\right. \\
& \left.\quad+16 \eta^{4}\left(1+\eta^{2}\right)\left(15 \phi^{4}+30 \pi^{2} \tau^{2} \phi^{2}+7 \pi^{4} \tau^{4}\right)\right\}+\frac{1}{16 \omega_{\perp}} \times \\
& \quad \times \sum_{k=1}^{\infty} \frac{(-1)^{k} e^{-k \phi / \tau}\{(\eta+1) \operatorname{sh}(k(\eta+1) / 2 \eta \tau)+(\eta-1) \operatorname{sh}(k(\eta-1) / 2 \eta \tau)\}}{\operatorname{sh}^{3}(k / 2 \tau) \operatorname{sh}^{2}(k / 2 \eta \tau)},
\end{aligned}
$$

осциллирующая составляющая $\widetilde{\Theta}_{x}^{\text {rig }}$ для иррациональных значений параметра $\eta$ определяется выражением

$$
\begin{aligned}
& \widetilde{\Theta}_{x}^{\mathrm{rig}}=\sum_{k=1}^{\infty}\left(A_{k}^{\mathrm{rig}} \cos (2 \pi k \phi)+B_{k}^{\mathrm{rig}} \sin (2 \pi k \phi)+C_{k}^{\mathrm{rig}} \cos (2 \pi k \eta \phi)+D_{k}^{\mathrm{rig}} \sin (2 \pi k \eta \phi)\right), \\
& A_{k}^{\mathrm{rig}}=\frac{(\eta+1) A_{k}^{+}+(\eta-1) A_{k}^{-}}{\omega_{\perp}}= \\
& =\frac{\pi \tau}{4 \eta^{2} \omega_{\perp} \sin (\pi k / \eta) \operatorname{sh}\left(2 \pi^{2} k \tau\right)}\left\{1-2 \eta^{2}\left(1+2 \pi^{2} \tau^{2}+2 \phi^{2}\right)+2\left(1-2 \eta^{2}\right) \operatorname{ctg}^{2} \frac{\pi k}{\eta}+\right. \\
& \left.+4 \pi \eta \tau \operatorname{cth}\left(2 \pi^{2} k \tau\right)\left[\left(1-\eta^{2}\right) \operatorname{ctg} \frac{\pi k}{\eta}+2 \pi \eta \tau \operatorname{cth}\left(2 \pi^{2} k \tau\right)\right]\right\}, \\
& B_{k}^{\mathrm{rig}}=\frac{(\eta+1) B_{k}^{+}+(\eta-1) B_{k}^{-}}{\omega_{\perp}}=\frac{\pi \tau \phi\left[\left(1-\eta^{2}\right) \operatorname{ctg}(\pi k / \eta)+2 \pi \eta \tau \operatorname{cth}\left(2 \pi^{2} k \tau\right)\right]}{\omega_{\perp} \eta \sin (\pi k / \eta) \operatorname{sh}\left(2 \pi^{2} k \tau\right)} \\
& C_{k}^{\mathrm{rig}}=\frac{(\eta+1) C_{k}^{+}+(\eta-1) C_{k}^{-}}{\omega_{\perp}}= \\
& =\frac{\pi \tau \eta(-1)^{k+1}\left[\left(2 \eta^{2}-1\right) \operatorname{ctg}(\pi k \eta)+2 \pi \eta^{2} \tau \operatorname{cth}\left(2 \pi^{2} k \eta \tau\right)\right]}{2 \omega_{\perp} \sin ^{2}(\pi k \eta) \operatorname{sh}\left(2 \pi^{2} k \eta \tau\right)}, \\
& D_{k}^{\mathrm{rig}}=\frac{(\eta+1) D_{k}^{+}+(\eta-1) D_{k}^{-}}{\omega_{\perp}}=\frac{(-1)^{k+1} \pi \tau \eta^{3} \phi}{\omega_{\perp} \sin ^{2}(\pi k \eta) \operatorname{sh}\left(2 \pi^{2} k \eta \tau\right)},
\end{aligned}
$$


а для случая рациональных значений параметра $\eta=m / n$

$$
\begin{aligned}
\widetilde{\Theta}_{x}^{\text {rig }}= & \sum_{k=1}^{\infty}\left(A_{k}^{\prime \text { rig }} \cos (2 \pi k \phi)+B_{k}^{\prime \text { rig }} \sin (2 \pi k \phi)+C_{k}^{\prime \text { rig }} \cos (2 \pi k \eta \phi)+D_{k}^{\prime \text { rig }} \sin (2 \pi k \eta \phi)\right), \\
A_{k}^{\text {rig }}= & \left\{\begin{array}{ll}
A_{k}^{\text {rig }}, & k / m \notin \mathbb{N}, \\
P_{k}^{\text {rig }}, & k / m \in \mathbb{N},
\end{array} \quad B_{k}^{\prime \text { rig }}= \begin{cases}B_{k}^{\text {rig }}, & k / m \notin \mathbb{N}, \\
Q_{k}^{\text {rig }}, & k / m \in \mathbb{N},\end{cases} \right. \\
C_{k}^{\text {rig }}= & \left\{\begin{array}{ll}
C_{k}^{\text {rig }}, & k / n \notin \mathbb{N}, \\
0, & k / n \in \mathbb{N},
\end{array} \quad D_{k}^{\prime \text { rig }}= \begin{cases}D_{k}^{\text {rig }}, & k / n \notin \mathbb{N}, \\
0, & k / n \in \mathbb{N},\end{cases} \right. \\
P_{k}^{\text {rig }}= & \frac{(-1)^{n k+1} \pi^{2} \tau^{2} \operatorname{cth}\left(2 \pi^{2} k \tau\right)}{6 \eta \omega_{\perp} \operatorname{sh}\left(2 \pi^{2} k \tau\right)}\left[1+\eta^{2}\left(2 \eta^{2}-1\right)-4 \eta^{2}\left(\eta^{2}+1\right)\left(3 \phi^{2}+5 \pi^{2} \tau^{2}\right)+\right. \\
& \left.+24 \pi^{2} \tau^{2} \eta^{2}\left(\eta^{2}+1\right) \operatorname{cth}^{2}\left(2 \pi^{2} k \tau\right)\right], \\
Q_{k}^{\text {rig }}= & \frac{(-1)^{n k+1} \pi \tau \phi}{6 \eta \omega_{\perp} \operatorname{sh}\left(2 \pi^{2} k \tau\right)}\left[1+\eta^{2}\left(2 \eta^{2}-1\right)-4\left(\eta^{2}+1\right)\left(\phi^{2}+3 \pi^{2} \tau^{2}\right)+\right.
\end{aligned}
$$

Снова приведем выражение для гладкой составляющей в пределе $\tau \rightarrow 0$ :

$$
\begin{aligned}
& \left.\bar{\Theta}_{x}^{\text {rig }}(\tau)\right|_{\tau=0}= \\
& \quad=\frac{7-21 \eta^{2}-44 \eta^{4}+24 \eta^{6}+120 \eta^{2}\left(-1+\eta^{2}-2 \eta^{4}\right) \phi^{2}+240 \eta^{4}\left(1+\eta^{2}\right) \phi^{4}}{2880 \omega_{\perp} \eta^{3}} \underset{ }{\eta} \underset{ }{\approx} \\
& \quad \approx \frac{240 \lambda^{4}-120 \lambda^{2}-17}{1440}+\frac{240 \lambda^{4}-360 \lambda^{2}+59}{8640}\left(\eta^{2}-1\right),
\end{aligned}
$$

а также приближенные выражения для коэффициентов $A_{k}^{\mathrm{rig}}, B_{k}^{\mathrm{rig}}, C_{k}^{\mathrm{rig}}, D_{k}^{\mathrm{rig}}$ вблизи сферического предела:

$$
\begin{array}{ll}
A_{k}^{\mathrm{rig}} \approx-\frac{\pi T}{\sin ^{3}(\pi k / \eta) \operatorname{sh}\left(2 \pi^{2} k T\right)}, & B_{k}^{\mathrm{rig}} \approx \frac{(-1)^{k} \pi T \lambda\left(1-\eta^{2}\right)}{\sin ^{2}(\pi k / \eta) \operatorname{sh}\left(2 \pi^{2} k T\right)}, \\
C_{k}^{\mathrm{rig}} \approx-\frac{\pi T}{\sin ^{3}(\pi k \eta) \operatorname{sh}\left(2 \pi^{2} k T\right)}, & D_{k}^{\mathrm{rig}} \approx \frac{(-1)^{k+1} \pi T \lambda}{\sin ^{2}(\pi k \eta) \operatorname{sh}\left(2 \pi^{2} k T\right)} .
\end{array}
$$

Из полученных формул мы снова замечаем, что коэффициенты $A_{k}^{\mathrm{rig}}, B_{k}^{\mathrm{rig}}, C_{k}^{\mathrm{rig}}$, $D_{k}^{\text {rig }}$ при приближении к сферическому пределу растут. Однако неограниченного увеличения вклада осциллирующей составляющей твердотельного момента инерции не происходит, и в сферическом пределе она принимает следующее предельное выражение для высоких значений $\lambda$ :

$$
\begin{gathered}
\left.\widetilde{\Theta}_{x}^{\text {rig }}(\eta)\right|_{\eta=1} \approx \sum_{k=1}^{\infty} \frac{(-1)^{k} 4 \pi^{2} T^{2} \lambda^{2}}{\operatorname{sh}^{2}\left(2 \pi^{2} k T\right)}\left[\operatorname{ch}\left(2 \pi^{2} k T\right) \cos (2 \pi k \lambda)+\right. \\
\left.+\frac{\lambda}{3 \pi T} \operatorname{sh}\left(2 \pi^{2} k T\right) \sin (2 \pi k \lambda)\right]
\end{gathered}
$$


Сравнивая выражения (57) и (47), заключаем, что амплитуды гармоник осциллирующей составляющей твердотельного момента инерции с ростом $k$ убывают быстрее, чем амплитуды гармоник в выражении (47), поэтому осцилляции твердотельного выражения для момента инерции выражены значительно слабее при приближении к сферическому пределу, чем осцилляции выражения (16) для момента инерции.

В пределе $T=0$ формула (57) принимает вид

$$
\begin{aligned}
\left.\widetilde{\Theta}_{x}^{\mathrm{rig}}\right|_{\eta=1, T=0} & \approx \sum_{k=1}^{\infty} \frac{(-1)^{k} \lambda^{2}}{\pi^{2} k^{2}}\left[\cos (2 \pi k \lambda)+\frac{2 \pi k \lambda}{3} \sin (2 \pi k \lambda)\right]= \\
& =\lambda^{2}\left[(\lambda-m)\left(\frac{\lambda}{3}-m\right)-\frac{1}{12}\right], \\
& m-\frac{1}{2}<\lambda<m+\frac{1}{2}, \quad m=0,1,2, \ldots,
\end{aligned}
$$

который не вырождается в гладкую форму, как в случае с выражением (16) для момента инерции. Поэтому твердотельный момент инерции в сферическом пределе при $T=0$ не исчезает, а осцилляции сохраняются. Для высоких значений $\lambda$ зависимость момента инерции $\left.\Theta_{x}^{\text {rig }}\right|_{\eta=1, T=0}$ приобретает вид “ступенчатой” функции

$$
\left.\left.\Theta_{x}^{\mathrm{rig}}\right|_{\eta=1, T=0} \simeq \Theta_{x, \mathrm{TF}}^{\mathrm{rig}}\right|_{\eta=1, \lambda=m}=\frac{m^{2}}{6}, \quad m-\frac{1}{2}<\lambda<m+\frac{1}{2}, \quad m=0,1,2, \ldots
$$

\section{7. ЗАКЛЮЧЕНИЕ}

В настоящей работе проведен строгий аналитический расчет зависимости момента инерции нагретой конечной системы нуклонов от химического потенциала в рамках модели принудительного вращения с потенциалом анизотропного гармонического осциллятора. Для расчета была использована стандартная методика преобразования Меллина, которая позволила разделить момент инерции на две составляющие: гладкую и осциллирующую. Полученные явные аналитические выражения для указанных составляющих, которые справедливы во всем интервале температур и параметров деформации ядра, позволяют непосредственно (без обращения к численным расчетам) выделить важные характерные особенности зависимостей момента инерции конечной ферми-системы от числа частиц, температуры и деформации системы. В частности, показано, что осцилляции момента инерции в зависимости от химического потенциала усиливаются при приближении к сферическому пределу, когда $\omega_{x, y} \rightarrow \omega_{z}$, а с увеличением температуры быстро спадают. При нулевой температуре в сферическом пределе, так же как и в гидродинамической модели вращения ядра, момент инерции обращается в нуль. Проведен расчет твердотельного момента инерции, который реализуется при выполнении условия статистически равновесного вращения, и показано, что осцилляции в зависимости от химического потенциала присутствуют и здесь. Однако осцилляции для твердотельного момента инерции проявляются слабее и не исчезают при нулевой температуре в сферическом пределе. 
Развитая в работе методика разделения физических величин на гладкую и осциллирующую составляющие и их аналитического расчета может быть успешно применена и к другим системам, где проявляется сложное немонотонное поведение физических характеристик, обусловленное ограниченностью геометрии системы или движения частиц.

\section{Список литературы}

[1] D. R. Inglis, Phys. Rev., 96:4 (1954), 1059-1065; 97:3 (1955), 701-706; 103:6 (1956), 1786-1795.

[2] A. Bohr, B. R. Mottelson, Mat.-Fys. Medd. Dan. Vid. Selsk., 30:1 (1955).

[3] A. Bohr, B. Mottelson, Nuclear Structure, v. 2, Benjamin, New York, 1975.

[4] В. В. Пашкевич, С. Фраундорф, ЯФ, 20 (1974), 1122.

[5] И.Н. Михайлов, К. Неергор, В.В.Пашкевич, С. Фрауендорф, ЭЧАЯ, 8:6 (1977), 1338-1411.

[6] M. Brack, R. K. Bhaduri, Semiclassical Physics, Frontiers in Physics, 96, Westview Press, Boulder, CO, 2003.

[7] K. Bencheikh, P. Quentin, J. Bartel, Nucl. Phys. A, 571:3 (1994), 518-540.

[8] E. Chabanat, J. Meyer, K. Bencheikh, P. Quentin, J. Bartel, Phys. Lett. B, 325:1-2 (1994), $13-19$.

[9] V. M. Strutinsky, Nucl. Phys. A, 95:2 (1967), 420-442; 122:1 (1968), 1-33.

[10] M. Brack, L. Damgaard, A.S. Jensen, H. C. Pauli, V. M. Strutinsky, C. Y. Wong, Rev. Modern Phys., 44:2 (1972), 320-406.

[11] В. Г. Зелевинский, ЯФ, 22:6 (1975), 1085-1095.

[12] L. Jacak, W. Nawrocka, R. G. Nazmitdinov, A. Wojs, J. Phys. G, 21:9 (1995), 1205-1216.

[13] A. G. Magner, A. S. Sitdikov, A. A. Khamzin, J. Bartel, Phys. Rev. C, 81:6 (2010), 064302, $20 \mathrm{pp}$.

[14] M. J. Gutzwiller, J. Math. Phys., 12:3 (1967), 343-358.

[15] В. М. Струтинский, А. Г. Магнер, ЭЧАЯ, 7:2 (1976), 356-418.

[16] A. G. Magner, A.S. Sitdikov, A. A. Khamzin, J. Bartel, A. M. Gzhebinsky, Phys. Atom. Nucl., 73:8 (2010), 1398-1404.

[17] A. G. Magner, A. M. Gzhebinsky, A.S. Sitdikov, A. A. Khamzin, J. Bartel, Internat. J. Modern Phys. E, 19:4 (2010), 735-746.

[18] А.Р. Миннуллин, Д. А. Таюрский, Писъма в ЖЭТФ, 72:12 (2000), 891-897.

Поступила в редакцию 21.11.2012, после доработки 11.02.2013 